\title{
BMJ Open Influence of dysregulated expression of circular RNA on the diagnosis and prognosis of breast cancer in Asia: a meta-analysis study
}

\author{
Fengyuan Liu (D) , ${ }^{1}$ Xinrui Wu, ${ }^{1}$ Huixia Zhu, ${ }^{1}$ Feng Wang ${ }^{2,3}$
}

To cite: Liu F, Wu X, Zhu H, et al. Influence of dysregulated expression of circular RNA on the diagnosis and prognosis of breast cancer in Asia: a meta-analysis study. BMJ Open 2021;11:e044267. doi:10.1136/ bmjopen-2020-044267

- Prepublication history and additional supplemental material for this paper are available online. To view these files, please visit the journal online (http://dx.doi.org/10.1136/ bmjopen-2020-044267).

Received 29 August 2020 Accepted 01 October 202

Check for updates

(c) Author(s) (or their employer(s)) 2021. Re-use permitted under CC BY-NC. No commercial re-use. See rights and permissions. Published by BMJ.

${ }^{1}$ Department of Clinical Medicine, Medical School of Nantong University, Nantong University, Nantong, China ${ }^{2}$ Department of Laboratory Medicine, Nantong University Affiliated Hospital, Nantong,

China

${ }^{3}$ Department of Laboratory Medicine, Public Health School of Nantong University, Nantong University, Nantong, China

Correspondence to

Professor Huixia Zhu; zhuhuixiantu@163.com

\section{ABSTRACT}

Objective Recent studies have reported a correlation between non-coding RNAs such as circular RNAs (circRNAs) and clinical value of various cancers. However, the diagnostic and prognostic role of circRNA in breast cancer remains controversial.

Design Systematic review and meta-analysis.

Methods Diagnostic efficacy was estimated by sensitivity, specificity and area under the curve (AUC). Pooled HRs with $95 \%$ Cls estimated overall survival (OS), and ORs with 95\% Cls investigated clinical features.

Results By searching PubMed, Embase, Web of Science, CNKI and Cochrane Library, we obtained a total of 29 studies with 4405 patients. A shorter survival time was associated with high expression levels of tumourpromoter circRNAs (OS: $\mathrm{HR}=2.43,95 \% \mathrm{Cl} 2.20$ to 2.92 , $\mathrm{p}<0.001)$, and tumour-suppressor circRNAs were related to a favourable prognosis (OS: $\mathrm{HR}=0.32,95 \% \mathrm{Cl} 0.23$ to $0.44, p<0.001)$. Furthermore, high expression levels of oncogenic circRNAs were associated with poor clinical outcomes; tumour-suppressor circRNAs showed the opposite result. As for the diagnostic role, the outcome indicated an AUC of $0.82(95 \% \mathrm{Cl} 0.78$ to 0.85$)$, with $85 \%$ sensitivity and $86 \%$ specificity to distinguish patients with breast cancer from healthy controls.

Conclusion Dysregulated expression of circRNA was related to diagnosis and prognosis in breast cancer, which indicated it might be a novel biomarker and a target of therapy for breast cancer.

PROSPERO registration number CRD42020207912.

\section{INTRODUCTION}

In the twenty-first century, breast cancer is one of the malignant cancers in developed and developing countries. ${ }^{12}$ Mortality from breast cancer ranked third in all cancers in 2018, according to the latest data from Global Cancer Statistics. ${ }^{3}$ Currently, owning to the increasing incidence of breast cancer, new methods are needed to improved diagnostic accuracy and therapeutic effect of breast cancer. Therefore, many researchers spend significant effort searching for novel biomarkers which predict the progression
Strengths and limitations of this study

- This study reported the guidelines of the Metaanalysis Of Observational Studies in Epidemiology group and Preferred Reporting Items for Systematic reviews and Meta-analysis statement.

- Analyses have been undertaken respecting potential sources of known statistical heterogeneity.

- This meta-analysis to describe on the association of circular RNAs expression with breast cancer prognosis and diagnostic features, which indicated it might be a novel biomarker and target of therapy for breast cancer.

- The variability in methods of assessing risk and reporting of frequency of risk characteristics limited analyses.

of breast cancer, in terms of early diagnosis, prognosis and treatment.

Circular RNAs (circRNAs) are a special kind of endogenous non-coding RNAs, with a closed covalent ring structure connecting $3^{\prime}$ and $5^{\prime}$ ends. ${ }^{45}$ They are also competitive RNAs that, along with long-chain non-coding RNAs, co-regulate microRNAs. ${ }^{6}$ CircRNA participates in the growth and development of cancer, diabetes, nervous system disorders, cardiovascular diseases, and other diseases through various biological roles, such as sponge action, protein translation and binding protein action. $^{7-9}$ Recently, a growing number of studies showed that numerous circRNAs have been discovered and have a close relation with the development of breast cancer. ${ }^{4}$ It is well known that the function of circRNA has great potential in metastasis, invasion, initiation and carcinogenesis of breast cancer. However, the role of circRNA in breast cancer remains controversial based on existing research. Therefore, we conducted this meta-analysis to summarise their diagnostic and prognostic role in breast cancer. 


\section{MATERIALS AND METHODS}

\section{Search strategy}

Based on the guidelines of the Meta-analysis Of Observational Studies in Epidemiology group and Preferred Reporting Items for Systematic Reviews and Meta-analysis s0 ${ }^{10}$ we searched the Web of Science, EMBASE, PubMed, Cochrane Library and CNKI databases up to 1 August 2020. The searching items were: ('circRNA' or 'circular RNA' or 'has_circ') and ('breast cancer' or 'breast neoplasms' or 'mammary cancer' or 'breast tumour'). To avoid missing documents, we manually screened the reference lists of the retrieved articles.

\section{Eligibility criteria}

Eligible articles conformed to the following criteria: (1) The subjects were patients with breast cancer confirmed by histopathological diagnosis and the clinical data were complete; (2) The article evaluated the relationship between circRNA expression and clinicopathological features, diagnosis and prognosis; and (3) It was a case-control study. The exclusion criteria were: (1) The subjects of the study were not human; (2) The publication was not a primary research publication (eg, a review, correspondence, repeated publication, conference summary). (3) There were no data available in the article.

\section{Quality assessment}

The quality of primary diagnostic studies was assessed by the QUADAS-2 tool. The QUADAS tool consists of four key domains, including patient selection, index test, reference standard and flow of patients. The answer of risk for bias could be rated as 'no' (0 points), 'yes' (one point) or 'unclear' (0 points). ${ }^{11}$ The Newcastle-Ottawa Scale was used to evaluate the quality of case-control studies from three aspects: selection, comparability and results. ${ }^{12}$ Publications below six points were considered as low quality; high quality was above six points.

\section{Data extraction}

Two researchers (FL, HZ) separately evaluated the suitability of all retrieved studies and extracted the relevant data. The two researchers contacted a third researcher (XW) when there was a disagreement. The following data were extracted: (a) Title, first author, ethnicity, year of publication, cancer type, patient size, circRNA signature, follow-up (months); (b) Expression status of circRNA, pooled HRs, detection methods, overall survival (OS) and their corresponding 95\% CIs; (c) Sensitivity, specificity and area under the curve (AUC) of circRNAs for diagnosis; (d) Clinical data with age, menopause, tumour size, TNM stage, lymph node metastasis, oestrogen receptor, progesterone receptor (PR) and human epidermal growth factor receptor-2 (HER-2).

\section{Statistical analysis}

HRs and 95\% CIs were used to estimate OS. Sensitivity, specificity and AUC were involved in the diagnostic analysis. Clinical parameters were assessed using ORs and 95\% CIs. Heterogeneity was assessed by the $\chi^{2}$ test and $\mathrm{I}^{2}$ index.
High heterogeneity was judged with an $\mathrm{I}^{2}$ value $>50 \%$. Subgroup and sensitivity analyses were performed to investigate potential sources of heterogeneity when $\mathrm{I}^{2}>50 \%$. Publication bias was evaluated quantitatively using Deek's funnel plot, Begg's tests and Egger's tests. Statistical analyses were performed by Revman V.5.3 and Stata V.15.1 software (Stata Corporation, College Station, Texas, USA).

\section{Patient and public involvement}

No patients or the public were involved in the research.

\section{Ethics approval statement}

This study did not involve human participants.

\section{RESULTS}

\section{Selection of studies}

A total of 366 articles were initially obtained from the databases and other sources based on keywords (figure 1). Among these articles, 186 duplicate articles were removed, and 180 articles remained. By looking through titles and abstracts, 65 articles were left for further full-text review. We then reviewed the full texts of these articles carefully and excluded an additional 36 articles. Finally, 29 articles $^{13-41}$ were included in this meta-analysis, including 21 studies for clinicopathological feature ${ }^{15-35} 8$ for diagnosis ${ }^{13-17}$ and 26 for prognosis. ${ }^{17-41}$

\section{Characteristics of included studies and quality assessment}

The study characteristics are shown in tables 1-2. A total of 4405 patients with breast cancer from Asia were collected from the 29 included articles. The publication years ranged from 2017 to 2020 . The follow-up period varied from 40 months to 200 months. According to their function in breast cancer, 24 circRNAs were recognised as tumour promoters/upregulated and 11 were tumour suppressors/downregulated. With the QUADAS-II criteria, the scores of all diagnostic researches were $\geq 4$ (online supplemental figure 1). Assessed by the NewcastleOttawa Scale, the points of the prognostic trials were $\geq 6$ (table 3). The scores suggested that all of the included articles are of high quality.

\section{Overall survival}

The OS was reported in 27 studies. Elevated expression of tumour-suppressor circRNAs was related to a favourable prognosis $(\mathrm{HR}=0.32,95 \%$ CI 0.23 to $0.44, \mathrm{p}<0.001)$ (figure 2). A fixed-effect model was applied because there was low heterogeneity $\left(I^{2}=0 \%, p=0.429\right)$. Conversely, high expression of tumour-promoter circRNAs was associated with an unfavourable prognosis $(\mathrm{HR}=2.43,95 \%$ CI 2.20 to $2.92, \mathrm{p}<0.001$ ) (figure 3 ). There was no significant heterogeneity $\left(I^{2}=0 \%, p=0.791\right)$, so the fixed-effect model was performed for this analysis as well.

\section{Diagnostic analysis}

The outcomes of pooled sensitivity and specificity were shown in figure 4 . The summary estimates are as follows: 


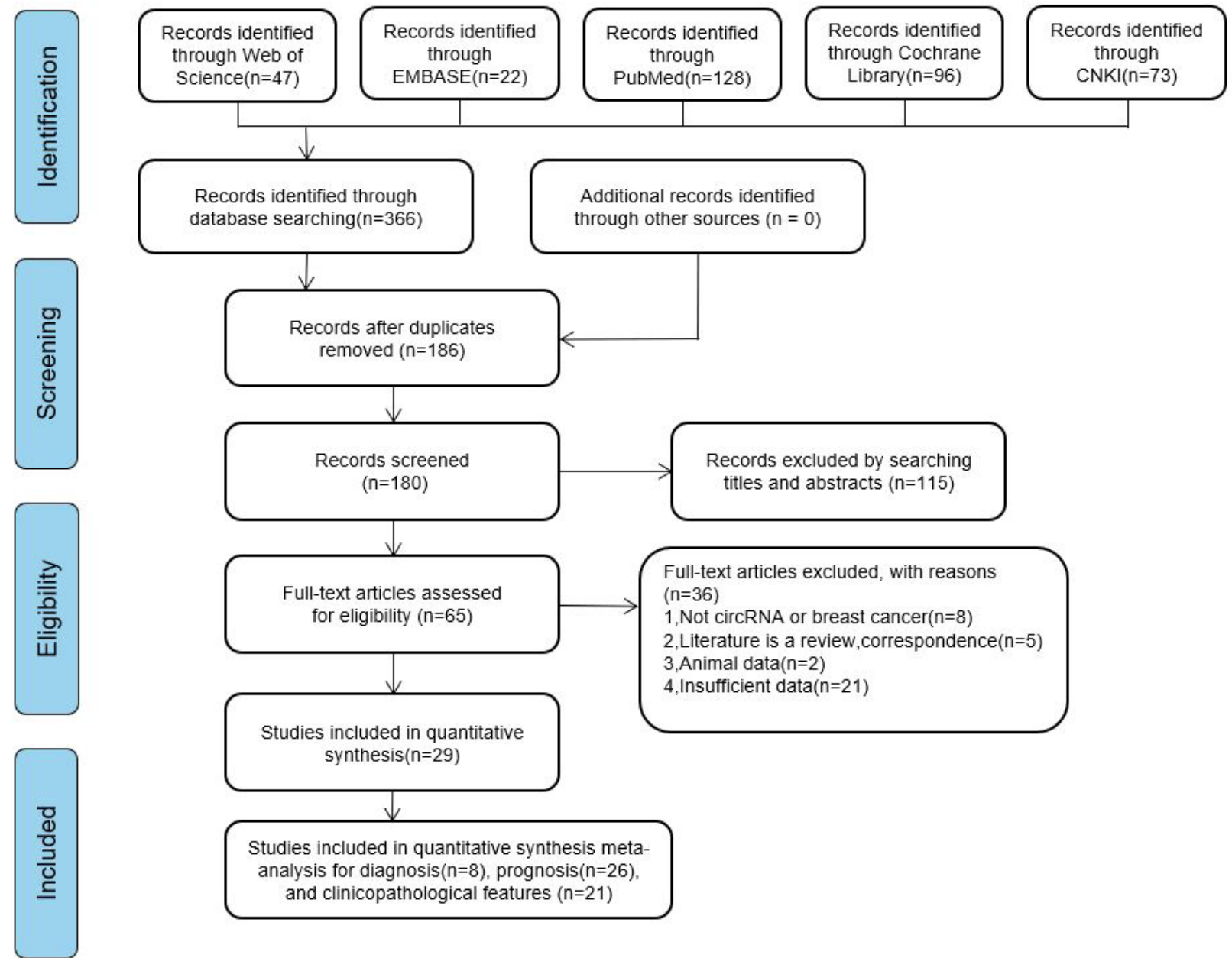

Figure 1 Data acquisition and screening flow chart. circRNA, circular RNA.

specificity, 0.76 (95\% CI 0.62 to 0.86$)$; sensitivity, 0.75 (95\% CI 0.66 to 0.82 ); negative likelihood ratio, 0.33 (95\% CI 0.21 to 0.50$)$; positive likelihood ratio, $3.10(95 \%$ CI 1.80 to 5.60); and overall diagnostic OR, 10.0 (95\% CI 4.0 to 26.0 ). Besides, a summary receiver operator characteristic curve was carried out in figure 5 and AUC was $0.82(95 \%$ CI 0.78 to 0.85$)$. A significant heterogeneity was detected in the pooled sensitivity $\left(\mathrm{I}^{2}=86.07 \%\right)$ and specificity $\left(I^{2}=85.35 \%\right)$. To explore the potential source of heterogeneity, we did subgroup analysis according to sample size, year, ethnicity, expression state of circRNA. Finally, sample size was the main source of heterogeneity. As shown in online supplemental figure 2, the heterogeneity was reduced in the pooled sensitivity $\left(\mathrm{I}^{2}=2.46 \%\right)$ and

Table 1 Main characteristics of studies for diagnostic analysis

\begin{tabular}{|c|c|c|c|c|c|c|c|c|c|}
\hline \multirow[b]{2}{*}{ Study } & \multirow[b]{2}{*}{ Year } & \multirow[b]{2}{*}{ circRNA signature } & \multicolumn{2}{|c|}{ Sample size } & \multirow[b]{2}{*}{ Detection methods } & \multirow[b]{2}{*}{ Expression status } & \multicolumn{3}{|c|}{ Diagnostic power } \\
\hline & & & Case & Control & & & Sen & Spe & AUC \\
\hline Zheng et $a l^{17}$ & 2020 & circSEPT9 & 60 & 60 & qRT-PCR & Upregulated & 0.750 & 0.633 & 0.711 \\
\hline Li et $a l^{13}$ & 2019 & circ-VRK1 & 350 & 163 & qRT-PCR & Downregulated & 0.617 & 0.791 & 0.720 \\
\hline Yin et $a l^{16}$ & 2018 & hsa_circ_0001785 & 57 & 17 & qRT-PCR & Upregulated & 0.786 & 0.756 & 0.771 \\
\hline Yin et $a l^{16}$ & 2018 & hsa_circ_0068033 & 57 & 17 & qRT-PCR & Downregulated & 0.732 & 0.578 & 0.619 \\
\hline Lü et $a l^{14}$ & 2017 & hsa_circ_006054 & 51 & 51 & qRT-PCR & Upregulated & 0.650 & 0.690 & 0.710 \\
\hline Lü et $a l^{14}$ & 2017 & hsa_circ_100219 & 51 & 51 & qRT-PCR & Upregulated & 0.690 & 0.710 & 0.780 \\
\hline
\end{tabular}

AUC, area under the receiver operator characteristic curve; circRNA, circular RNA; qRT-PCR, quantitative real-time PCR; sen, sensitivity; spe, specificity. 


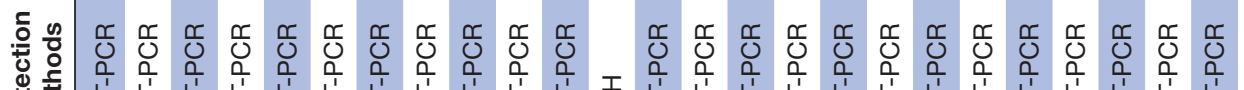

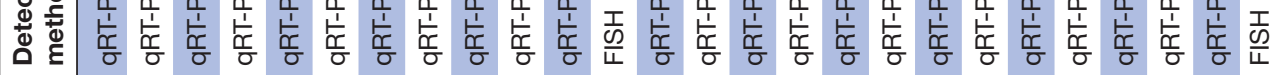

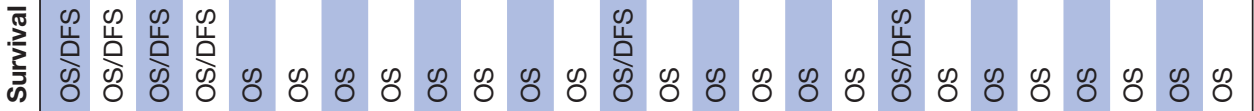

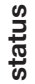

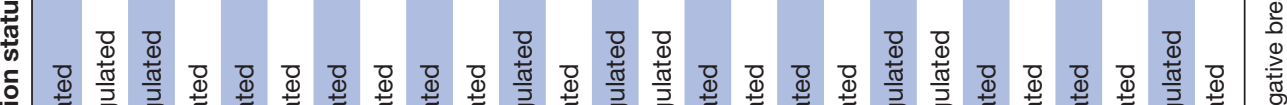

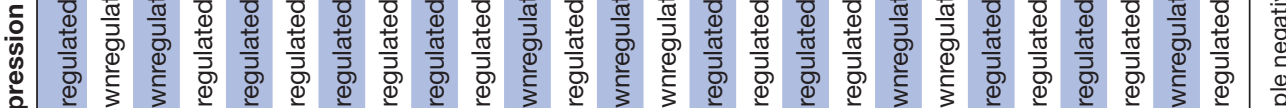

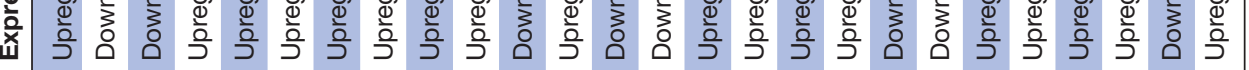

$\stackrel{\square}{\$}$

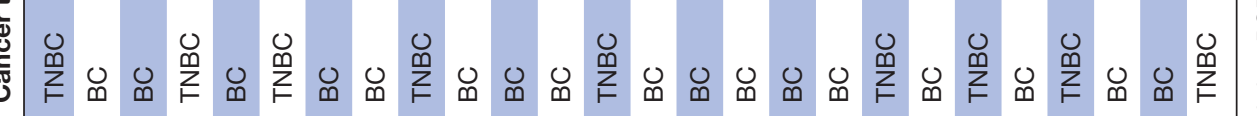

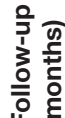

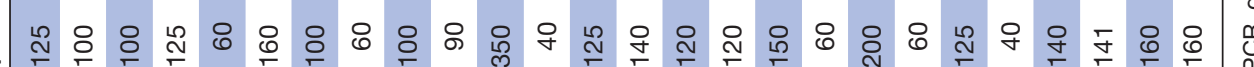

造

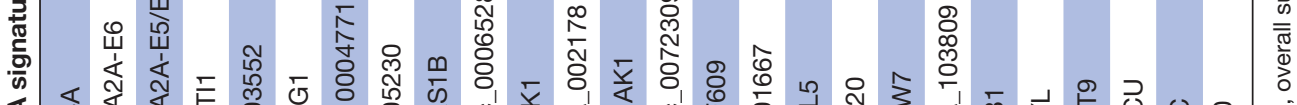

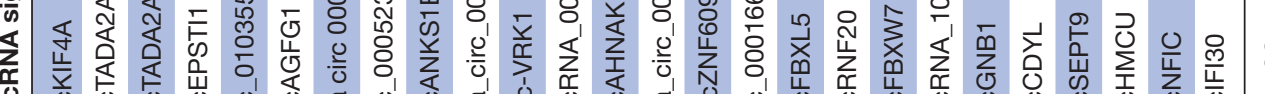

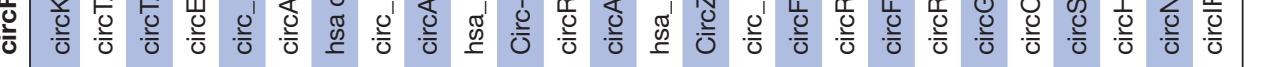

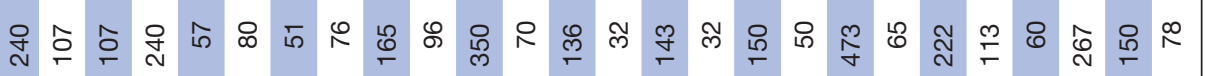
(5)

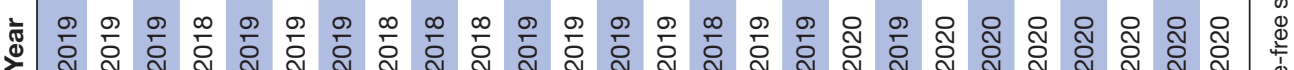

焉

旁

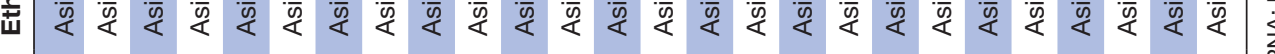

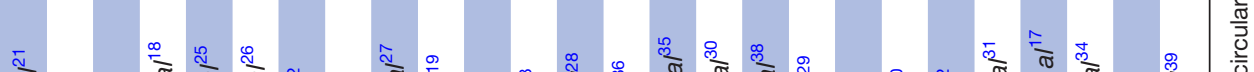

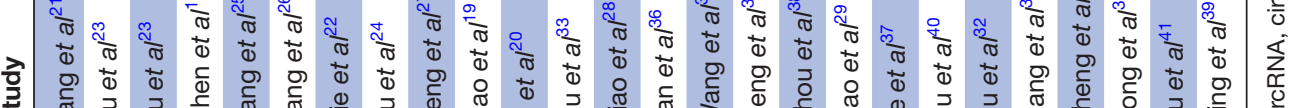

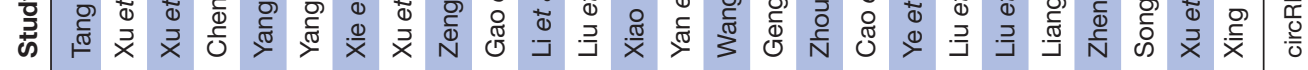


Table 3 Study quality assessed via the Newcastle-Ottawa Scale checklist

\begin{tabular}{|c|c|c|c|c|}
\hline Study & Selection & Comparability & Outcome & Total score \\
\hline Tang et $a l^{21}$ & thts & 计战 & 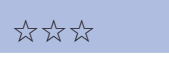 & 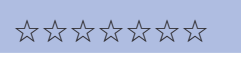 \\
\hline Xu et $a l^{23}$ & 论㐾 & 武论 & 论场 & 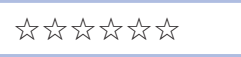 \\
\hline Chen et $a l^{18}$ & 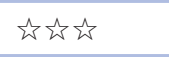 & $\hat{s}$ & 论㑔 & 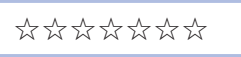 \\
\hline Yang et $a l^{25}$ & 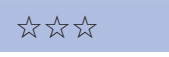 & $\vec{y}$ & 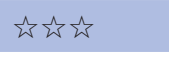 & 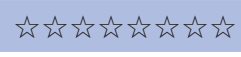 \\
\hline Xie et $a l^{22}$ & 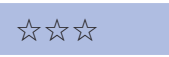 & 牙论 & $\sum \hbar$ & 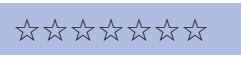 \\
\hline Xu et $a l^{24}$ & 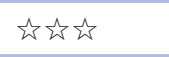 & 武败 & 论弥柁 & 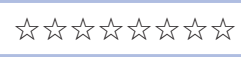 \\
\hline Zeng et $a l^{27}$ & tht & $\underline{t}$ & $\hbar \sqrt{2}$ & 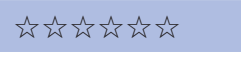 \\
\hline Gao et al ${ }^{19}$ & 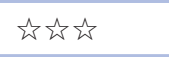 & 武许 & 论论㑔 & 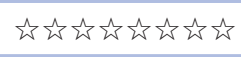 \\
\hline Yan et $a l^{36}$ & 论许 & 武许 & 论㑔 & 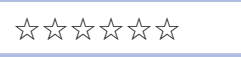 \\
\hline Wang et $\left.a\right|^{35}$ & 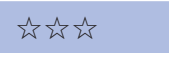 & $\hat{t} \hat{x}$ & 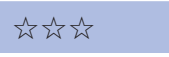 & 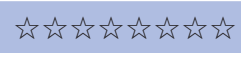 \\
\hline Geng et $a /^{30}$ & 论㑔 & 功㑔 & 论㑔 & 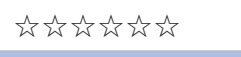 \\
\hline Zhou et $a l^{38}$ & 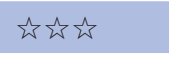 & $\hat{⿻ 心 ㇒}$ & 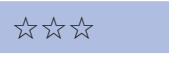 & 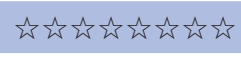 \\
\hline Cao et $a l^{29}$ & 弥㑔 & 为㑔 & 论㑔 & 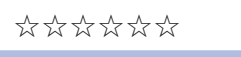 \\
\hline Ye et $\left.a\right|^{37}$ & 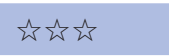 & 象站 & 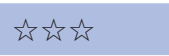 & 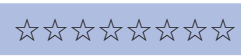 \\
\hline Liu et $\left.a\right|^{40}$ & 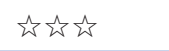 & 论弥 & 论放弥 & 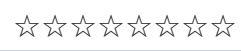 \\
\hline Liu et $\left.a\right|^{32}$ & 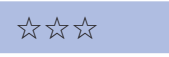 & 氙市 & 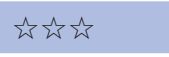 & 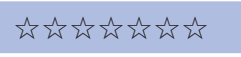 \\
\hline
\end{tabular}

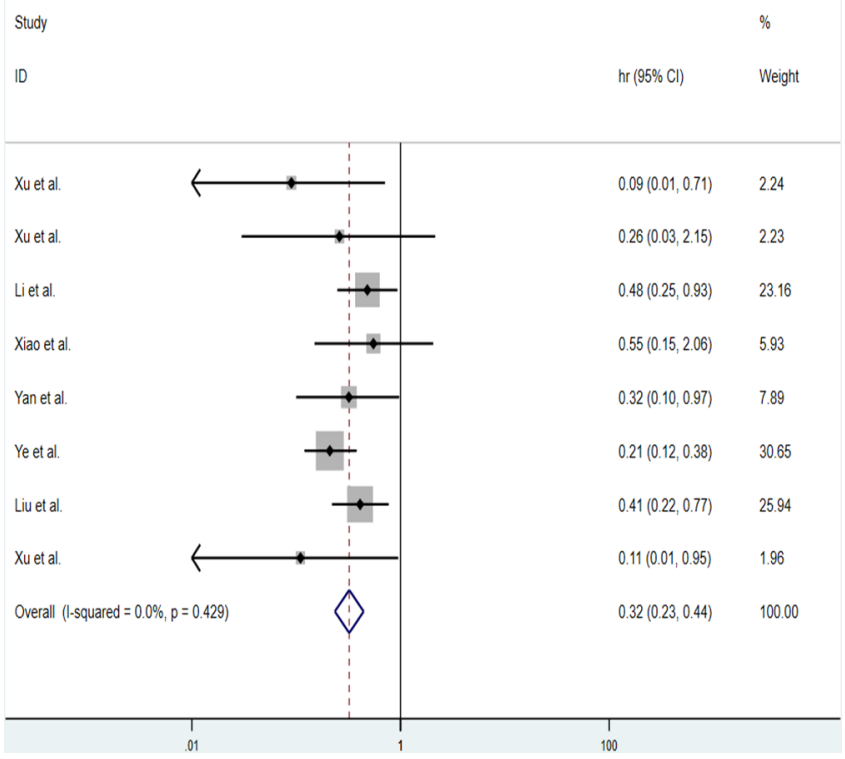

Figure 2 Forest plots for overall survival according to the type of tumour-suppressor circular RNA (circRNA). specificity $\left(\mathrm{I}^{2}=0.00 \%\right)$ after two large sample studies were excluded. The above outcomes suggested that circRNAs might be an ideal diagnostic biomarker for breast cancer.

\section{Clinicopathological association}

Twenty-one studies were included to evaluate the relationship between circRNA expression and the clinicopathological features of patients with breast cancer. As presented in table 4, prominent associations were observed. Elevated levels of tumour-promoter circRNAs were associated with adverse clinical outcomes, including tumour size $(\mathrm{OR}=2.84,95 \% \mathrm{CI} 2.07$ to $3.91, \mathrm{p}<0.001)$, TNM stage $(\mathrm{OR}=2.71,95 \%$ CI 2.00 to $3.67, \mathrm{p}=0.001)$, lymph node metastasis $(\mathrm{OR}=2.75,95 \% \mathrm{CI} 1.99$ to 3.75 , $\mathrm{p}<0.001)$, oestrogen receptor $(\mathrm{OR}=0.61,95 \%$ CI 0.43 to $0.87, \mathrm{p}=0.006)$ and HER2 $(\mathrm{OR}=0.60,95 \% \mathrm{CI} 0.39$ to 0.93 , $\mathrm{p}=0.022$ ). Elevated levels of tumour-suppressor circRNAs were negatively correlated to the clinical features: age $(\mathrm{OR}=0.66,95 \%$ CI 0.46 to $0.95, \mathrm{p}=0.024)$, tumour size ( $\mathrm{OR}=0.54,95 \%$ CI 0.36 to $0.80, \mathrm{p}=0.002)$, lymph node metastasis $(\mathrm{OR}=0.57,95 \%$ CI 0.39 to $0.83, \mathrm{p}=0.004)$, TNM 


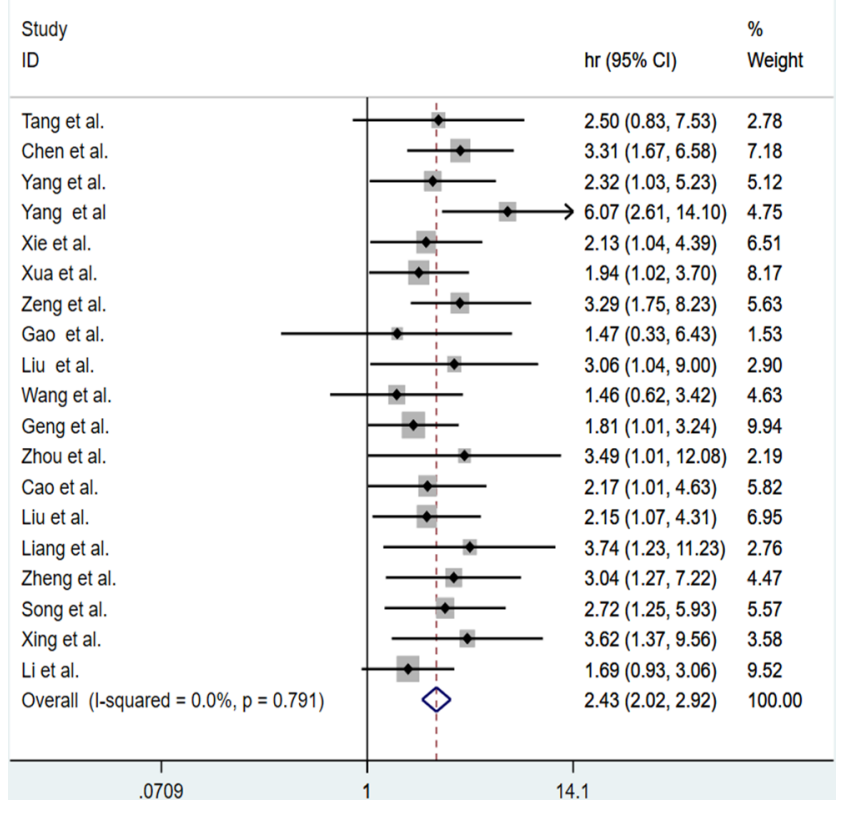

Figure 3 Forest plots for overall survival according to the type of oncogenic circular RNA (circRNA).

stage $(\mathrm{OR}=0.63,95 \%$ CI 0.45 to $0.90, \mathrm{p}=0.011)$ and HER2 $(\mathrm{OR}=0.50,95 \%$ CI 0.28 to $0.89, \mathrm{p}=0.019)$. No significant associations were found in terms of menopause or PR $(\mathrm{p}>0.05)$.

\section{Publication bias}

Judged by Deeks' funnel plot, there was no evidence of publication bias $(\mathrm{p}=0.66)$ in the diagnostic analysis (online supplemental figure 3). Begg's funnel plot (online supplemental figure 4, $\mathrm{p}=0.983$ ) and Egger's test (online supplemental figure $5, \mathrm{p}=0.937$ ) indicated that there was no clear publication bias in the analysis

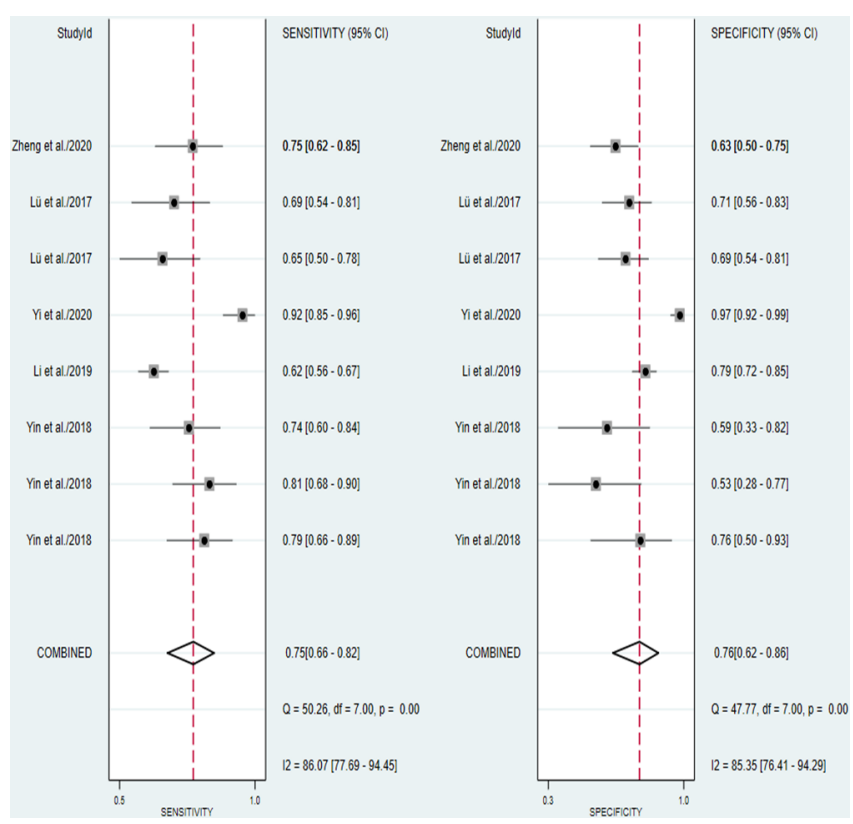

Figure 4 Forest plot of sensitivity and specificity of circular RNAs (circRNAs) for the diagnosis of breast cancer.

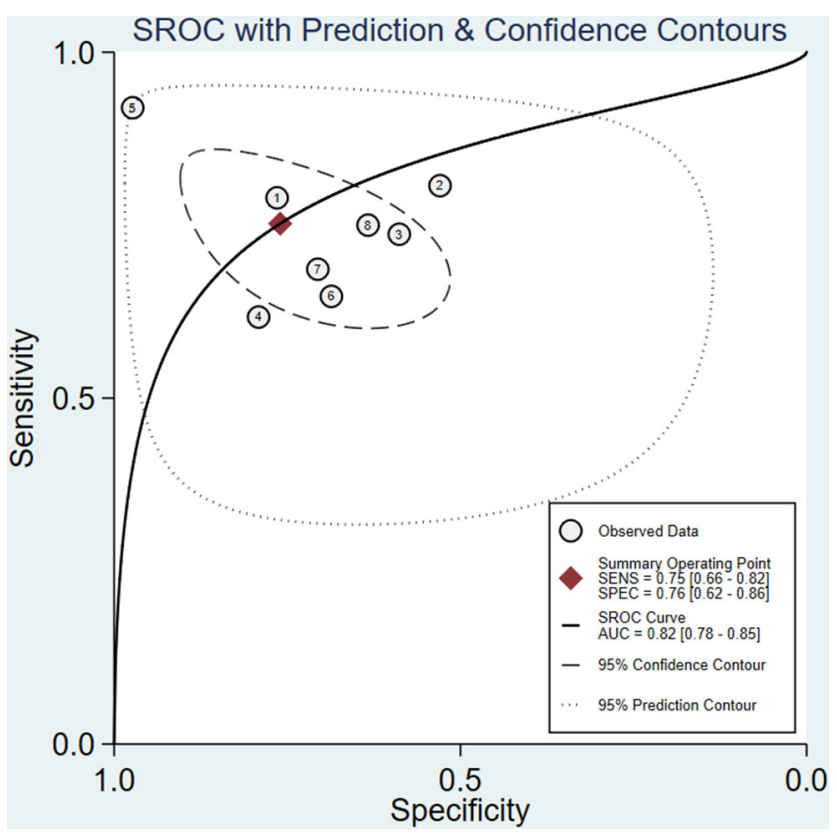

Figure 5 The summary receiver operator characteristic (SROC) curve. AUC, area under the ROC curve; ROC, receiver operator characteristic.

of circRNAs in terms of OS. These outcomes indicated that circRNAs are likely to be a favourable diagnostic and prognostic biomarker for breast cancer.

\section{DISCUSSION}

Up to now, plenty of predictors have been found and applied in the diagnosis and prognosis of breast cancer, including oestrogen receptor, HER2, BRCA and miRNA. Recently, circRNAs have been widely recommended due to their high conservation, high stability, high expression and specificity. ${ }^{5}$ CircRNA is recognised as a novel biomarker which has the potential to play a significant role in the development of breast cancer. For instance, Huang $e t a t^{42}$ and Huang $e t a t^{43}$ have summarised that circRNAs may act as important biomarkers for diagnosis and prognosis in lung cancer and osteosarcoma, respectively, by meta-analysis. Research into the role of circRNAs in breast cancer is increasing, but the clinical value of circRNAs is debatable. Current research discovered that circRNAs correlated with small tumour size, longer survival time and acted as antioncogenes in breast cancer. Whereas, more research proved that circRNAs might function as a vital oncogene for breast cancer. ${ }^{1-9}$ Based on clinical research, we conducted this meta-analysis to summarise the diagnostic and prognostic role of circRNA in breast cancer.

A total of 29 articles with 4405 patients with breast cancer in Asia were included in this study. According to circRNAs' function in breast cancer, we divided circRNAs into two groups. Some circRNAs such as circEPSTI1 were markedly upregulated in breast cancer and were considered as tumour-promoter circRNAs (tables 1-2). It is 
Table 4 Clinical characteristics of circRNAs in breast cancer

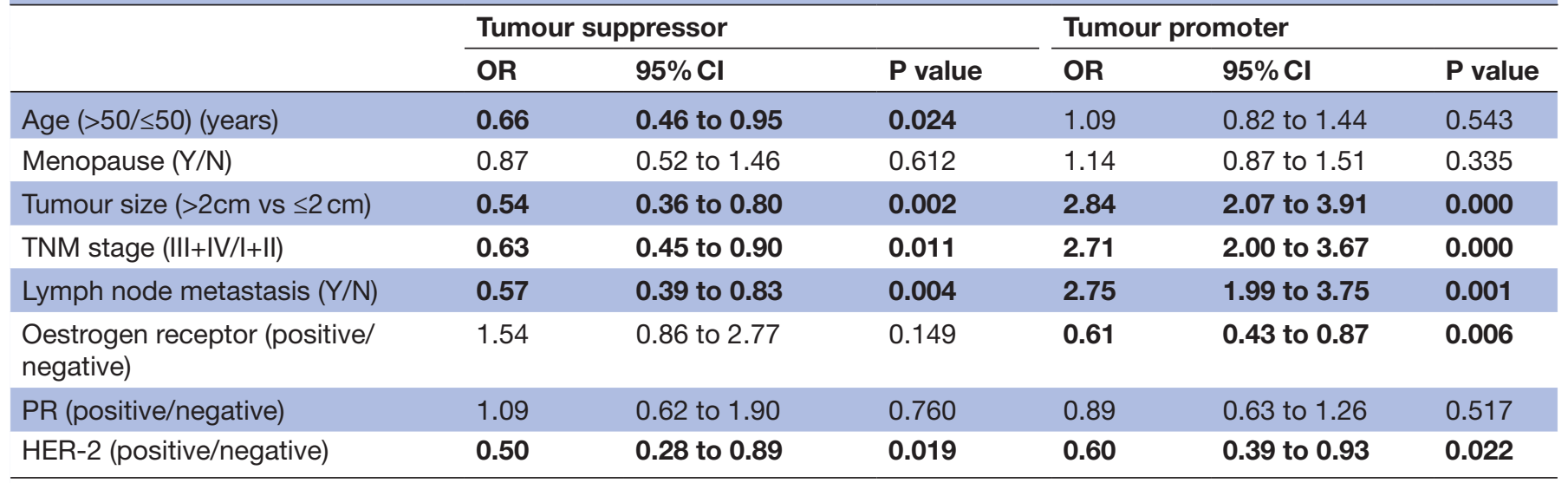

The results are in bold if $\mathrm{p}<0.05$.

circRNA, circular RNA; HER-2, human epidermal growth factor receptor-2; N, no; PR, progesterone receptor; Y, yes.

interesting that in breast cancer, no matter whether it is upregulation or downregulation, different biomarkers have the same effect through various mechanisms. For example, circSEPT9 is able to regulate expression of the leukaemia inhibitory factor (LIF) via sponging miR-637 and activating the LIF/Stat3 signalling pathway involved in progression of triple negative breast cancer (TNBC) ${ }^{17}$ besides, circEPSTI1 binds to miR-4753 and miR-6809 as a miRNA sponge to regulate BCL11A expression and affect TNBC proliferation and apoptosis. ${ }^{18}$ Opposite to this, the others were identified as tumour-suppressor circRNAs when circRNAs were downregulated in breast cancer (tables 1-2); hsa_circ_0068033 exerts biological functions by sponging miR-659, ${ }^{16}$ but circAHNAK1 acted as a miR-421 competitive endogenous RNA to attenuate the inhibitory effect of miR-421 on its target gene RASA1. ${ }^{28}$ In pooled analysis, high expression levels of oncogenic circRNAs were significantly associated with poor prognoses, whereas, evaluated tumour-suppressor circRNAs predicted favourable OS. Moreover, our study showed an AUC of 0.82 , with $75 \%$ sensitivity and $76 \%$ specificity, suggesting that circRNAs are good diagnostic markers for breast cancer. In terms of clinical features, evaluated oncogenic circRNA was also significantly related to bigger size of the tumour, higher rates of lymph node metastasis and higher TNM stage. Antioncogenic circRNA was opposite (table 4).

Despite the promising data, there are some limitations to our study. First, all the patients in our study were selected from an Asian population. Patients from other regions, such as Europe, were not included. The results of this study should be interpreted with caution. Second, the sample size in this study was small and more high-quality clinical studies are needed.

\section{CONCLUSION}

Dysregulated expression of circRNA was related to diagnosis and prognosis in breast cancer, which indicated it might be a novel biomarker and target of therapy for breast cancer.

Contributors Methodology: FL; XW; HZ. Formal analysis and investigation: FL; XW. Writing —original draft preparation: FL; XW. Writing—review and editing: FW. Funding acquisition: FW. Resources: FW. Supervision: HZ. HZ is the paper's guarantor.

Funding This work was supported by grants from the National Natural Science Foundation of China (Grant No: 81873978), Sixth Talent Peaks Project of Jiangsu Province (2018-WSW-068), Nantong University Student Innovation Programme (2020146).

Competing interests None declared.

Patient consent for publication Not applicable.

Provenance and peer review Not commissioned; externally peer reviewed.

Data availability statement Data are available in a public, open access repository. Data are available upon reasonable request. All data relevant to the study are included in the article or uploaded as supplementary information.

Supplemental material This content has been supplied by the author(s). It has not been vetted by BMJ Publishing Group Limited (BMJ) and may not have been peer-reviewed. Any opinions or recommendations discussed are solely those of the author(s) and are not endorsed by BMJ. BMJ disclaims all liability and responsibility arising from any reliance placed on the content. Where the content includes any translated material, BMJ does not warrant the accuracy and reliability of the translations (including but not limited to local regulations, clinical guidelines, terminology, drug names and drug dosages), and is not responsible for any error and/or omissions arising from translation and adaptation or otherwise.

Open access This is an open access article distributed in accordance with the Creative Commons Attribution Non Commercial (CC BY-NC 4.0) license, which permits others to distribute, remix, adapt, build upon this work non-commercially, and license their derivative works on different terms, provided the original work is properly cited, appropriate credit is given, any changes made indicated, and the use is non-commercial. See: http://creativecommons.org/licenses/by-nc/4.0/.

ORCID iD

Fengyuan Liu http://orcid.org/0000-0001-9333-9449

\section{REFERENCES}

1 Winters S, Martin C, Murphy D, et al. Breast cancer epidemiology, prevention, and screening. Prog Mol Biol Trans/ Sci 2017;151:1-32.

2 Anastasiadi Z, Lianos GD, Ignatiadou $\mathrm{E}$, et al. Breast cancer in young women: an overview. Updates Surg 2017;69:313-7.

3 Ferlay J, Colombet M, Soerjomataram I, et al. Cancer incidence and mortality patterns in Europe: estimates for 40 countries and 25 major cancers in 2018. Eur J Cancer 2018;103:356-87. 
4 Jahani S, Nazeri E, Majidzadeh-A K, et al. Circular RNA; a new biomarker for breast cancer: a systematic review. J Cell Physiol 2020;235:5501-10

5 Memczak S, Jens M, Elefsinioti A, et al. Circular RNAs are a large class of animal RNAs with regulatory potency. Nature 2013;495:333-8.

6 Salzman J, Gawad C, Wang PL, et al. Circular RNAs are the predominant transcript isoform from hundreds of human genes in diverse cell types. PLoS One 2012;7:e30733.

7 Zhou S-Y, Chen W, Yang S-J, et al. The emerging role of circular RNAs in breast cancer. Biosci Rep 2019;39:BSR20190621.

8 Lu D, Xu A-D, . Mini review: circular RNAs as potential clinical biomarkers for disorders in the central nervous system. Front Genet 2016;7:53.

9 Zhao Z, Li X, Jian D, et al. Hsa circ 0054633 in peripheral blood can be used as a diagnostic biomarker of pre-diabetes and type 2 diabetes mellitus. Acta Diabetol 2017;54:237-45.

10 Stroup DF, Berlin JA, Morton SC, et al. Meta-Analysis of observational studies in epidemiology: a proposal for reporting. meta-analysis of observational studies in epidemiology (moose) group. JAMA 2000;283:2008-12.

11 Whiting PF, Rutjes AWS, Westwood ME, et al. QUADAS-2: a revised tool for the quality assessment of diagnostic accuracy studies. Ann Intern Med 2011;155:529-36.

12 Deeks JJ, Dinnes J, D'Amico R, et al. Evaluating non-randomised intervention studies. Health Technol Assess 2003;7:iii-173.

$13 \mathrm{Li} \mathrm{Y,} \mathrm{Li} \mathrm{H.} \mathrm{Circular} \mathrm{RNA} \mathrm{VRK1} \mathrm{correlates} \mathrm{with} \mathrm{favourable} \mathrm{prognosis,}$ inhibits cell proliferation but promotes apoptosis in breast cancer. $J$ Clin Lab Anal 2020;34:e22980.

14 Lü L, Sun J, Shi P, et al. Identification of circular RNAs as a promising new class of diagnostic biomarkers for human breast cancer. Oncotarget 2017;8:44096-107.

15 Yi Z, Li Y, Wu Y, et al. Circular RNA 0001073 attenuates malignant biological behaviours in breast cancer cell and is delivered by nanoparticles to inhibit mice tumour growth. Onco Targets Ther 2020;13:6157-69.

16 Yin W-B, Yan M-G, Fang X, et al. Circulating circular RNA hsa circ_0001785 acts as a diagnostic biomarker for breast cancer detection. Clin Chim Acta 2018;487:363-8.

17 Zheng $X$, Huang M, Xing L, et al. The circRNA circSEPT9 mediated by E2F1 and elF4A3 facilitates the carcinogenesis and development of triple-negative breast cancer. Mol Cancer 2020;19:73.

18 Chen $B$, Wei $W$, Huang $X$, et al. circEPSTI1 as a prognostic marker and mediator of triple-negative breast cancer progression. Theranostics 2018;8:4003-15.

19 Gao D, Qi X, Zhang X, et al. hsa_circRNA_0006528 as a competing endogenous RNA promotes human breast cancer progression by sponging miR-7-5p and activating the MAPK/ERK signaling pathway. Mol Carcinog 2019;58:554-64.

$20 \mathrm{Li} \mathrm{Y,} \mathrm{Li} \mathrm{H.} \mathrm{Circular} \mathrm{RNA} \mathrm{VRK1} \mathrm{correlates} \mathrm{with} \mathrm{favourable} \mathrm{prognosis,}$ inhibits cell proliferation but promotes apoptosis in breast cancer. $J$ Clin Lab Anal 2020;34:e22980.

21 Tang $\mathrm{H}$, Huang $\mathrm{X}$, Wang J, et al. circKIF4A acts as a prognostic factor and mediator to regulate the progression of triple-negative breast cancer. Mol Cancer 2019;18:23.

22 Xie R, Tang J, Zhu X, et al. Silencing of hsa circ 0004771 inhibits proliferation and induces apoptosis in breast cancer through activation of miR-653 by targeting ZEB2 signaling pathway. Biosci Rep 2019;39:BSR20181919.

$23 \mathrm{Xu}$ J-Z, Shao C-C, Wang X-J, et al. circTADA2As suppress breast cancer progression and metastasis via targeting miR-203a-3p/ SOCS3 axis. Cell Death Dis 2019;10:175.
24 Xu Y, Yao Y, Leng K, et al. Increased expression of circular RNA circ_0005230 indicates dismal prognosis in breast cancer and regulates cell proliferation and invasion via miR-618/ CBX8 signal pathway. Cell Physiol Biochem 2018;51:1710-22.

25 Yang L, Song C, Chen Y, et al. Circular RNA circ_0103552 forecasts dismal prognosis and promotes breast cancer cell proliferation and invasion by sponging miR-1236. J Cell Biochem 2019;120:15553-60.

26 Yang $R$, Xing $L$, Zheng $X$, et al. The circRNA circAGFG1 acts as a sponge of miR-195-5p to promote triple-negative breast cancer progression through regulating CCNE1 expression. Mol Cancer 2019;18:4.

27 Zeng K, He B, Yang BB, et al. The pro-metastasis effect of circANKS1B in breast cancer. Mol Cancer 2018;17:160.

28 Xiao W, Zheng S, Zou Y, et al. CircAHNAK1 inhibits proliferation and metastasis of triple-negative breast cancer by modulating miR-421 and RASA1. Aging 2019;11:12043-56.

29 Cao L, Wang M, Dong Y, et al. Circular RNA circRNF20 promotes breast cancer tumorigenesis and Warburg effect through miR-487a/ HIF-1 $\alpha /$ HK2. Cell Death Dis 2020;11:145

30 Geng Z, Wang W, Chen H, et al. Circ_0001667 promotes breast cancer cell proliferation and survival via Hippo signal pathway by regulating TAZ. Cell Biosci 2019;9:104.

31 Liang G, Ling Y, Mehrpour M, et al. Autophagy-Associated circRNA circCDYL augments autophagy and promotes breast cancer progression. Mol Cancer 2020;19:65

32 Liu P, Zou Y, Li X, et al circGNB1 facilitates triple-negative breast cancer progression by regulating miR-141-5p-IGF1R axis. Front Genet 2020;11:193.

33 Liu T, Ye P, Ye Y, et al. Circular RNA hsa_circRNA_002178 silencing retards breast cancer progression via microRNA-328-3p-mediated inhibition of COL1A1. J Cell Mol Med 2020;24:2189-201.

34 Song $X$, Liang $Y$, Sang $Y$, et al. circHMCU promotes proliferation and metastasis of breast cancer by sponging the let- 7 family. Mol Ther Nucleic Acids 2020;20:518-33.

35 Wang S, Xue X, Wang R, et al. CircZNF609 promotes breast cancer cell growth, migration, and invasion by elevating p70S6K1 via sponging miR-145-5p. Cancer Manag Res 2018;10:3881-90.

36 Yan L, Zheng M, Wang H. Circular RNA hsa_circ_0072309 inhibits proliferation and invasion of breast cancer cells via targeting miR492. Cancer Manag Res 2019;11:1033-41.

37 Ye F, Gao G, Zou Y, et al. circFBXW7 inhibits malignant progression by sponging miR-197-3p and encoding a 185-aa protein in triplenegative breast cancer. Mol Ther Nucleic Acids 2019;18:88-98.

38 Zhou $\mathrm{H}$, Tang $\mathrm{G}$, Zhao M, et al. circFBXL5 promotes breast cancer progression by sponging miR-660. J Cell Mol Med 2020;24:356-61.

39 Xing L, Yang $\mathrm{R}$, Wang $\mathrm{X}$, et al. The circRNA circlFI30 promotes progression of triple-negative breast cancer and correlates with prognosis. Aging 2020;12:10983-1003.

40 Liu M, Luo C, Dong J, et al. CircRNA 103809 suppresses the proliferation and metastasis of breast cancer cells by sponging MicroRNA-532-3p (miR-532-3p). Front Genet 2020;11:485.

41 Xu G, Ye D, Zhao Q, et al. circNFIC suppresses breast cancer progression by sponging miR-658. J Cancer 2020;11:4222-9.

42 Huang $X$, Zhang W, Shao Z. Prognostic and diagnostic significance of circRNAs expression in lung cancer. $J$ Cell Physiol 2019;234:18459-65.

43 Huang X, Yang W, Zhang Z, et al. Dysregulated circRNAs serve as prognostic and diagnostic markers in osteosarcoma by sponging microRNA to regulate the downstream signaling pathway. J Cell Biochem 2020;121:1834-41. 